\title{
Atomic Force Microscopy as a Tool to Assess the Specificity of Targeted Nanoparticles in Biological Models of High Complexity
}

\section{Carla P. Gomes, Cátia D. F. Lopes, Michael Leitner, Andreas Ebner, Peter Hinterdorfer, and Ana P. Pêgo*}

C. P. Gomes, Dr. C. D. F. Lopes, Prof. A. P. Pêgo

INEB - Instituto de Engenharia Biomédica

i3S - Instituto de Investigação e Inovação em Saúde

Rua Alfredo Allen 208, 4200-135 Porto, Portugal

E-mail: apego@ineb.up.pt

C. P. Gomes, Prof. A. P. Pêgo

Faculdade de Engenharia da Universidade do Porto

R. Dr. Roberto Frias

4200-465 Porto, Portugal

Dr. C. D. F. Lopes

Faculdade de Medicina da Universidade do Porto

Alameda Prof. Hernâni Monteiro

4200-319 Porto, Portugal

Dr. M. Leitner, Prof. A. Ebner, Prof. P. Hinterdorfer Institute of Biophysics

Johannes Kepler University

Gruberstraße 40, 4020 Linz, Austria

Prof. A. P. Pêgo

ICBAS - Instituto de Ciências Biomédicas Abel Salazar

Universidade do Porto

Rua de Jorge Viterbo Ferreira 228, 4050-313 Porto, Portugal

Originally published in Advanced Healthcare Materials. 2017 Nov;6(21). doi: 10.1002/adhm.201700597. Epub $2017 \mathrm{Jul} 28$.

"This is the peer reviewed version of the following article: Gomes CP, Lopes CDF, Leitner M, Ebner A, Hinterdorfer P, Pego AP. Atomic Force Microscopy as a Tool to Assess the Specificity of Targeted Nanoparticles in Biological Models of High Complexity. Adv Healthc Mater. 2017;6(21), which has been published in final form at https://doi.org/10.1002/adhm.201700597. This article may be used for non-commercial purposes in accordance with Wiley Terms and Conditions for Use of Self-Archived Versions." 


\section{ABSTRACT}

The ability to design nanoparticle delivery systems capable of selectively target their payloads to specific cell populations is still a major caveat in nanomedicine. One of the main hurdles is the fact that each nanoparticle formulation needs to be precisely tuned to match the specificities of the target cell and route of administration. In this work, molecular recognition force spectroscopy (MRFS) is presented as a tool to evaluate the specificity of neuron - targeted trimethyl chitosan nanoparticles to neuronal cell populations in biological samples of different complexity. The use of atomic force microscopy tips functionalized with targeted or non - targeted nanoparticles made it possible to assess the specific interaction of each formulation with determined cell surface receptors in a precise fashion. More importantly, the combination of MRFS with fluorescent microscopy allowed to probe the nanoparticles vectoring capacity in models of high complexity, such as primary mixed cultures, as well as specific subcellular regions in histological tissues. Overall, this work contributes for the establishment of MRFS as a powerful alternative technique to animal testing in vector design and opens new avenues for the development of advanced targeted nanomedicines.

Keywords: atomic force spectroscopy, histological tissue samples, nanoparticle design, primary co-cultures, targeted nanomedicines

\section{INTRODUCTION}

One of the biggest challenges in medicine is to improve our ability to efficiently and precisely deliver therapeutic molecules to a desired target. The mean by which a drug is delivered could have a significant impact on its efficacy. The majority of drugs present an optimal concentration range within which maximum benefit is derived, and concentrations above or below these limits will more likely produce toxicity, having no therapeutic advantage.1 Another critical issue consists in the remarkable difficulty of many therapeutic molecules in overcoming biological barriers. 2 In this regard, the emergence of nanomedicine opens new therapeutic opportunities for bioactive molecules that cannot be used effectively as conventional drug formulations.3 Vectorizing these molecules by means of nanoparticle (NP) based structures has revolutionized the field of drug formulation and delivery, as these carrying systems present unique tunable physicochemical properties that allow to protect drugs from degradation and clearance, prevent harmful side - effects and improve bioavailability by providing controlled or timed drug release.4 Moreover, the attractiveness of these nanomaterials is that they can be also designed for targeted drug delivery at a specific disease site, improving the uptake of their payload and safety profile, ultimately resulting in an increased therapeutic index.5

In the last decades, research in nanomedicine has strongly focused on the use of specific ligands to be conjugated onto the NPs surface when aiming at increasing NP selectivity and specificity. 6 Nevertheless, the fact that each NP formulation needs to be precisely tuned to match the specificities of the target cell population and route of administration is time consuming and implies cumbersome testing, both in vitro and in vivo. Thus, technologies that enable the screening and characterization of a large number of candidates under biologically relevant conditions are still eagerly awaited.7

In this context, atomic force microscopy (AFM) has emerged as a powerful characterization platform, providing valuable insights that may have major implications for targeted NP design and 
development.8 AFM is a well - known high - resolution surface topography technique with nanometer resolution under physiological conditions. Furthermore, in addition to topographical imaging, the AFM cantilever offers the possibility to be used as a biosensing system, as it can be functionalized with different (bio)structures to probe their interactions with specific receptors, either isolated, reconstituted or in native biological samples. As a result, AFM has evolved from a mere physical microscopy technique into a standard method in pharmaceutical and life sciences, becoming a multifunctional molecular toolkit.8, 9

Taking advantage of this functionality, our group has previously proposed the use of molecular recognition force spectroscopy (MRFS) as a screening tool for the optimization of the density of targeting moieties toward improving receptor - specific binding of NPs to neuronal cells in simple cell culture systems.10 It remained to be established if the proposed methodology could be applied in models of higher complexity and biological relevance, to expand the impact and applicability of the technique.

In this work, we aimed at testing the specificity of gene carrying trimethyl chitosan (TMC) NPs actively targeted toward neuronal populations in complex biological models, that include in vitro heterogeneous primary neural cell cultures and innervated histological tissue sections, by MRFS. To promote the NP selective targeting toward neuronal cells, TMC NPs functionalized with the non toxic and neurotropic carboxylic $C$ - terminal fragment of the tetanus toxin heavy chain $(H C)$, with proven efficacy both in vitro11, 12 and in vivo,13, 14 were used.

To the best of our knowledge, this is the first report showing the application of force spectroscopy using primary cultures composed of mixed cell populations and/or histological tissue sections for the evaluation of NP vectoring capacity. We believe that our data validate MRFS as a valuable tool for the design and characterization of the targeting potential of new formulations that ultimately will lead to the appearance of more robust and advanced nanomedicines, regardless of the desired application and biological target. Furthermore, MRFS can be assumed as a promising alternative screening method to animal experimentation.

\section{Results}

\subsection{Polymer Characterization}

TMC with an average number molecular weight (urn:xwiley:dummy:media:adhm201700597:adhm201700597-math-0001) of $43.3 \mathrm{kDa}$, a degree of acetylation of $15.7 \%$ and a quaternization degree of $30.1 \%$ was used.15 To attain nanoparticle neurotropism, our group has been exploring the tethering of a pegylated HC fragment at the NPs surface.12-14, 16 Consequently, thiol groups were introduced into the TMC polymer backbone (TMCSH) by a previously described method.12, 14 During TMCSH complexation with plasmid DNA, the presence of thiol pending groups further contributes to the crosslinking of the polycation, stabilizing the resulting complexes, and allows the subsequent functionalization of the NPs. The total amount of thiol groups grafted to the polymer was $149.1 \pm 15.8 \mu \mathrm{mol} g-1$, as determined by the Ellman's assay, 17 corresponding to $4.7 \pm 0.5 \%$ substitution of TMC primary amines by thiol groups.

\subsection{Nanoparticle Preparation and Characterization}


Polymer - plasmid DNA binary complexes, hereafter referred to as naked NPs (Figure 1a), were prepared by coacervation, as a result of the electrostatic interaction between the positively charged polymer and the negatively charged plasmid DNA (pDNA). After NP stabilization at room temperature (RT) for $20 \mathrm{~min}$, the NPs were further functionalized by attachment of a $5 \mathrm{kDa}$ poly(ethylene glycol) (PEG) chains with a terminal methyl oxide group (Figure 1 b) or HC - PEG conjugates (Figure 1c), to yield non - targeted (nT) or targeted (Tg) NPs, respectively. Transmission electron microscopy images of each formulation are presented in (Figure $1 d-f$ ). To confirm the presence of the non - toxic carboxylic fragment of the tetanus toxin $(\mathrm{HC})$ at the NP surface, the $\mathrm{Tg}$ NPs were functionalized with HC modified with Odot $705 \mathrm{~nm}$ ITK carboxyl quantum dots (Invitrogen; further details in the Experimental Section) - hereafter designated as HC - quantum dots (QDs).

After preparation, all NPs were characterized in terms of mean average diameter size, polydispersity index (Pdl) and zeta - potential (surface charge) by dynamic light scattering. The ability of the developed NPs to efficiently condense pDNA was also measured, using a method previously setup by us.15 The obtained data from the NP characterization (Table 1 ) show that naked NPs presented significant higher average sizes $(>220 \mathrm{~nm}$ ) when compared to the functionalized ones $(<180 \mathrm{~nm})$. In agreement, the Pdl values were also significant higher $(p<0.05)$ for the naked NPs, when compared to the other formulations. This effect is ascribed to the presence of the PEG spacer at the surface of functionalized NPs, that contributes to the observed reduction of particle size.18 Regarding the zeta potential, the addiction of the PEG molecules to the NPs surface led to a reduction in the NP zeta potential between +2 and $+5 \mathrm{mV}$, depending on the presence or absence of the $\mathrm{HC}$ protein, respectively. Since $\mathrm{HC}$ is a positively charged protein at the $\mathrm{pH}$ of the NP preparation $(\mathrm{pH} 7.4)$, the slight higher zeta potential value of the Tg NPs in relation to the nT ones was to be expected. Regarding the NPs ability to condense pDNA, all formulations presented condensation efficiencies above $91 \%$, showing that NPs functionalization did not alter their ability to maintain their cargo.

\subsection{Nanoparticle Specificity Toward Neuronal versus Non - Neuronal Cell Lines}

The HC fragment is responsible for the tetanus toxin neurospecific binding to polygangliosides present at the neuronal cellular membrane (being GT1b the most predominant) that leads to the consequent toxin internalization and retrograde transport.19 First, the binding specificity of the purified $\mathrm{HC}$ fragment toward selected neuronal (ND7/23) and non - neuronal cell lines ( $\mathrm{RN} 22$ and $\mathrm{NIH}$ ${ }_{3} T_{3}$ ) was determined to confirm the validity of the chosen cell lines as in vitro models for the determination of the Tg NPs neurospecificity. The HC protein was modified with quantum dots ( $\mathrm{HC}$ QDs) and incubated with the three selected cell lines at $4{ }^{\circ} \mathrm{C}$ to allow ligand-receptor interaction but inhibit protein internalization. The obtained results (Figure 2) show that the HC - ODs specifically bind to the neuronal cell line (ND7/23) (Figure 2a), as no interaction was observed between the HC QDs and the control lines of schwannoma ( $\mathrm{RN}_{22}$ ) and fibroblasts ( $\mathrm{NIH}_{3} \mathrm{~T}_{3}$ ) cells (Figure $2 \mathrm{~b}_{1} \mathrm{c}$, respectively). To further confirm the specificity of the binding of the HC - ODs to the neuronal line, a preincubation of cells with a 100 - fold excess of unlabeled $\mathrm{HC}$ was performed prior to the incubation with the $\mathrm{QD}$ modified $\mathrm{HC}$, resulting in a significant drop of the observed fluorescent signal (data not shown). These results are in accordance with our previous findings, in which we have compared the $\mathrm{HC}$ binding profile to $\mathrm{ND}_{7} / 23$ and $\mathrm{NIH}_{3} \mathrm{~T}_{3}$ cell lines using $\mathrm{HC}$ labeled with rhodamine.11 After validation of the selected cell lines, we proceeded with the MRFS studies to assess the 
specificity of the different developed NP formulations toward the cell lines under testing. A heterobifunctional PEG18 linker was used to tether both, the HC fragment (via an acetal group) (Figure 2d) and the NPs (via a maleimide group) (Figure 2e) to an AFM tip. Representative forcedistance curves (FDC) obtained for naked, nT or Tg NPs in ND7/23 cells are shown in Figure $2 f-h$. When performing MRFS with naked NPs, independently of the cell line, the occurrence of multiple unbinding events was observed. These result from nonspecific low force $(<20 \mathrm{pN})$ electrostatic interactions established between the positively charged NPs (Table 1, zeta - potential data) and the negatively charged cell membrane. In contrast, most of the FDC obtained with the nT NPs did not present any unbinding events. On the other hand, the nT NPs are coated with PEG molecules, which offer a stealth effect and avert the interaction of these NPs with the cellular membrane. Consequently, most of the FDC resulting from the interaction of these NPs with cells did not present unbinding events of any kind (unspecific adhesion forces, electrostatic interactions or specific events), independently of the cell line tested. The same result was observed in the FDC obtained with the Tg NPs probed against the non - neuronal cell lines. Unsurprisingly, the acquisition of FDC with Tg NPs in ND7/23 cells presented single molecule unbinding events, comparable to those obtained with the HC - functionalized AFM tips (data not shown). Such occurrences were followed by a distinctive nonlinear force signal due to the elastic extension of the PEG linker present at the AFM tip surface, which allowed for the distinction of specific rupture events from unspecific adhesions. 20 The analysis of specific rupture events in a dataset of hundreds of FDC yields a force histogram that can be represented as a calculated probability density function (PDF), according to Baumgartner et al., allowing the determination of the most probable rupture force for the ligand-receptor pair.21 Representative PDFs for the interaction between $\mathrm{HC}$ and the different cell lines under testing are shown in Figure $2 \mathrm{i}$. The interaction between $\mathrm{HC}$ and $\mathrm{ND} 7 / 23$ cells presented a monomodal distribution peak around $30 \mathrm{pN}$ with a binding probability (BP) of $16.6 \%$. In contrast, the interaction of the $\mathrm{HC}$ fragment with the non - neuronal cell lines ( $\mathrm{RN}_{22}$ and $\mathrm{NIH}_{3} \mathrm{~T}_{3}$ ) resulted in a multimodal distribution with a low empirical probability density function and a BP value of $3.5 \%$ and $4.8 \%$, respectively. To attest that the measured forces resulted from the specific ligand-receptor pair interaction, ND7/23 cells surface receptors were blocked using free $\mathrm{HC}(1 \mathrm{mg} \mathrm{mL}-1)$ prior acquisition of FDC, which resulted in a significant fall of the BP from $16.6 \%$ to $4.4 \%(p<0.001)$. In Figure $2 j$ is presented the PDF graphic resulting from the $\mathrm{Tg}$ and $\mathrm{nTNPs}$ probing against the ND7/23 cell line. The $\mathrm{Tg}$ NPs presented a distribution peak similar to the one obtained for the $\mathrm{HC}$ fragment, with a comparable BP value (17.8\%). After cell surface blockage with free $\mathrm{HC}$, this value significantly decreased to $5.8 \%$, proving the specificity of this interaction. On the other hand, the use of AFM tips holding nT NPs yielded a statistically significant lower BP value (3.7\%) when compared to the tips with Tg NPs. The different BP values given from the measurement of the interactions between both $\mathrm{Tg}$ and $\mathrm{nT}$ NPs with the different cell lines is showed in Figure $2 \mathrm{k}$, each dot corresponding to the acquisition of $\approx 2000$ FDC in a given cell. Despite the statistically significant difference observed in the BP between these two NP systems toward the neuronal cell line, no statistical differences were observed between the two formulations in the non - neuronal cell lines. Moreover, the BP of the $\mathrm{Tg}$ NPs is significantly higher for the neuronal cell line than the non - neuronal cells $(p<0.001)$.

Finally, to support the data obtained through the MRFS studies, we assessed the internalization profile of these formulations by flow cytometry. Briefly, cells were incubated with the different TMC - based NPs containing YOYO ${ }^{\circledR}$ - 1 labeled pDNA for $2 \mathrm{~h}$ and afterward the percentage of cells with internalized NPs was quantified (Figure $2 \mathrm{l}$ ). In order to remove the external fluorescent signal (adsorbed NPs), cells were incubated with trypan blue and washed before FACS analysis. The obtained results were in line with the MRFS data, showing a significant decrease in the internalization 
of the Tg NPs for both non - neuronal cell lines ( $\mathrm{RN}_{22}$ and $\mathrm{NIH}_{3} \mathrm{~T}_{3}$ ), when compared to the naked NP formulation ( $p<0.001$ and $p<0.05$, respectively). Moreover, while the incubation with the nT NPs led to a decrease in the internalization extent, independently of the cell line tested, the Tg NPs were internalized into a high percentage of neuronal cells $(>90 \%)$, confirming their specific tropism toward this cell line.

\subsection{Nanoparticle Specificity for Neurons in Heterogeneous Primary Neuronal Cultures}

Following the determination of the binding specificity of the developed NPs in single - cell culture systems, we further proceeded our studies in an in vitro model of higher complexity using primary dissociated dorsal root ganglia (DRG) mixed cell cultures. These cultures are composed by the heterogeneous collection of cells that is present in the sensory ganglia that lie in the intervertebral foramina. Importantly, the culture reflects the diverse population of cells that constitute a DRG and the isolated sensory neurons retain features observed in vivo (e.g., the capacity to extend neurites). Besides DRG neurons, they are mainly constituted by Schwann cells, satellite cells and fibroblasts.

The $\mathrm{HC}$ fragment binding affinity and natural tropism to this mixed cell population was investigated using the previously modified HC - ODs. For neuronal cell identification, DRGs were co - labeled with the anti - $\beta$ III tubulin antibody (pan - neuronal marker). All cellular nuclei present in the culture were identified by $4^{\prime}, 6$ - Diamidino - 2 - Phenylindole (DAPI) staining. Representative images are shown in Figure $3 \mathrm{a}-\mathrm{d}$. One can observe the preferential binding of HC - QDs to neuronal cell bodies (white arrows), as well as neuronal axons, with negligible labeling being observed around the nuclei of non - neuronal cells. We next proceeded with the determination of the specificity of the HC fragment and the developed NP formulations toward the cells present in the DRG cultures by MRFS studies. By combining fluorescent microscopy with AFM, one was able to distinguish neuronal ( $\beta$ III tubulin positive cells) from non - neuronal cells and to locate the AFM tip above the cell type that we wanted to probe. The data obtained from the acquisition of FDC in both neuronal and non - neuronal cells with AFM tips functionalized with either the HC fragment, $\mathrm{nT}$ and Tg NPs is depicted in Figure 3e. As observed with the cell lines, FDC acquired with tips bearing naked NPs presented multiple bindings resulting from nonspecific interactions (data not shown). The BP obtained following the interaction between HC - functionalized AFM tips and neuronal cells was of $29 \%$, which was significantly higher than the value attained in the non - neuronal cells $(16 \% ; \mathrm{p}<0.001)$. Similar results were obtained for the AFM tips functionalized with Tg NPs, once again proving the targeted vectoring capacity of these NPs. AFM tips functionalized with nT NPs presented a BP of $11 \%$, independently of the cell type being tested $(p>0.05)$.

Representative PDF for the interaction between the nT or Tg NPs and specific cells from DRG cultures is shown in Figure $3 f$. Except for the interaction between Tg NPs with neuronal cells from DRGs, all tested conditions showed a multimodal distribution of forces with low binding probabilities. Contrariwise, measurements between Tg NPs and neuronal cells resulted in the appearance of three peaks in the force PDF, having as the most probable force $30 \mathrm{pN}$ with two other force peaks around 60 and $90 \mathrm{pN}$. For this ligand-receptor pair, most of FDCs present single rupture events, although multiple consecutive rupture events also occurred. Figure $3 \mathrm{~g}$ shows the distribution of rupture events per curve for a representative dataset of a neuronal cell probed with an AFM tip functionalized with Tg NPs, having a total BP of $26.7 \%$. A maximum of 3 consecutive events were observed, although the majority of the FDC with unbinding events only presented one event per curve $(\approx 70 \%)$.

.


To validate these data, we further assessed the cellular association profile of the developed NPs in primary DRG cultures by confocal microscopy (Figure 4). NPs loaded with YOYO ${ }^{\circledR}-1$ - labeled pDNA were incubated with cells for $2 \mathrm{~h}$. Afterward, cells were stained for $\beta$ III tubulin and were incubated with a dye that stained the cytoplasm and nuclei of all cells in culture - the HCS CellMaskTM (Thermo Fisher Scientific). This approach allowed for the distinction of NPs preferentially associated to neurons or to non - neuronal cell types. The obtained results corroborated the previous findings attained by MRFS. From the analysis of the images, one can observe that the naked NPs associate to all types of cells, irrespective of their origin. On the other hand, the nT NPs had minimal association to any cell type present in culture, explained by the stealth effect of the PEG molecules on the NP surface. As determined by MRFS, the Tg NP presented preferential tropism to the DRG neurons, confirming their specificity toward this cell type.

\subsection{Nanoparticle Targeting Capacity Toward Specific Cellular Regions in Histological Sections}

The expression and exposure of cellular surface receptors is strongly influenced not only by the cell type but also by the cell - cell crosstalk and the extracellular microenvironment. For that reason, the extrapolation of the results from NP characterization and uptake studies in vitro to an in vivo scenario is neither easy nor straightforward. In this context, we hypothesized that AFM could be a suitable tool to investigate the targeting capacity of NPs in a model that more closely resembles the in vivo scenario and, therefore, expected to be more clinically relevant. So, we next evaluated the interaction between the developed NPs and histological tissue sections. To the best of our knowledge, this is the first time that force spectroscopy studies with NPs were performed in this type of samples.

As our team is working toward the design of gene delivery vectors capable to target peripheral nerves after a minimal invasive peripheral administration (Figure 5a), we next assessed the binding specificity of the developed NPs toward histological sections of the innervated gastrocnemius muscle (site of administration) and the sciatic nerve (final target). Moreover, by taking advantage of the use of fluorescence microscopy combined with AFM, we were able to precisely position the AFM tip to perform MRFS in the subcellular regions of interest. After analyzing the biding profile of HC - ODs in the histological tissue sections (Figure $5 \mathrm{~b}, \mathrm{c}$ ), a broad labeling was observed in the sciatic nerve cryosection, as expected. Contrariwise, in the gastrocnemius muscle sections, HC - ODs fluorescent signal was only found at the neuromuscular junctions (NMJ) in colocalization with the $\beta$ III tubulin staining, with no signal being detected in the muscle tissue.

We then performed MRFS in determined cellular sub - populations of the tissue samples. FDC using NP - functionalized AFM tips were acquired in the sciatic nerve (regions labeled for $\beta$ III tubulin), muscle tissue (no labeling) and NMJ (positive for $\beta I I I$ and the postsynaptic marker $\alpha$ - bungarotoxin). The comparison between the BP of HC - , nT - , or Tg NPs - functionalized tips in the referred cellular regions are presented in Figure $5 \mathrm{~d}$. Both $\mathrm{HC}$ and Tg NPs tips presented comparable results ( $\mathrm{p}$ $>0.05$ ), with an average BP between $20 \%$ and $26 \%$ in the sciatic nerve. These values were identical to the ones previously obtained with the cellular models here tested (ND7/23 cells and primary neurons), confirming these as good models for testing the specificity of neuron - target NPs. Interestingly, these values were significantly higher $(p<0.001)$ in the NMJ, with a BP between $50 \%$ and $55 \%$, and significantly dropped to a BP around $8 \%$ in the case of muscle tissue probing $(p<0.001)$. 
Once again, the BP of the nT NPs did not significantly differ among the different cellular regions being tested (8-13\%). Moreover, after analysis of the PDF from the interaction between $\mathrm{nT}$ and Tg NPs in the sciatic nerve (Figure $5 \mathrm{e}$ ) and in the NMJ (Figure ${ }_{5} \mathrm{f}$ ), one can observe that only the Tg NPs present monomodal peak distributions, with higher force probabilities around 30-40 pN. Moreover, in the FDC acquired with Tg NPs tips in the NMJ a maximum of 6 consecutive events were observed contrary to the FDC from the sciatic nerve were the occurrence of multiple events was significantly lower (Figure $5 g)(p<0.001)$.

\subsection{Loading Rate Dependence for HC- or Nanoparticle-Receptor Complexes in Models of Different Complexity}

The most probable rupture force obtained for the different datasets (showed in the PDF histograms) is not a unitary value, as it depends on the time scale of the measurement.22 A receptor-ligand bond present a limited duration that is even shorter when an external force is applied to the bond, owing to thermal activation. In fact, the thermal energy of the solution also makes bonds break, which is essential for rendering possible time - controlled regulation of ligand - stimulated processes. 23 When a bond is pulled faster than its spontaneous dissociation rate, the ligand-receptor unbinding force depends not only on the molecules themselves but also on the loading rate established for the experiment.8, 24 In the case of a single energy barrier, the unbinding force rises with the logarithm of the loading rate in a linear fashion, yielding the natural kinetic off - rate constant (koff) that defines the lifetime of the bond in absence of any applied force, as well as the energetic barrier length scale $(x ß)$. To determine the loading rate dependence of both the $\mathrm{HC}$ fragment and the $\mathrm{HC}$ functionalized NPs (Tg NPs) in the biological models under testing, unbinding forces were evaluated at various retraction velocities (300-2000 $\mathrm{nm} \mathrm{s}-1)$. The most probable rupture forces were then plotted against the logarithm of the actual loading rate $(r)$. The loading rate was then computed by multiplying the retraction velocity by the effective spring constant (keff), which can be inferred from the slope of the FDC at rupture. 25

The loading rate dependence for the interaction between HC or Tg NPs in ND7/23 cells, primary cell cultures or tissue histological samples are shown in Figure $6 a-c$, respectively. For each dataset, a continuous curve with a linear slope was found, fitting the described theory. The obtained values of $x \beta$ and koff did not significantly varied among the tested conditions (Table 2$)(p>0.05)$. The fact that both $\mathrm{HC}$ or $\mathrm{HC}$ - functionalized NPs present identical rupture forces, independently of whether they were tested in cells or tissue samples, indicates that the functionalization of these NPs did not alter the binding kinetics between $\mathrm{HC}$ and its cellular receptors, and moreover, that no significance differences were observed among the different models tested.

\section{Discussion}

Tailored drug - delivery systems have become increasingly important in the development of novel medical therapies and pharmaceutical products.8 Interestingly, regardless of the substantial preclinical research efforts into developing actively targeted drug delivery systems, these technologies are still lacking in approved products and in clinical trials. 26

In addition to providing high - resolution topographical images of sample surfaces, AFM can be used to explore receptor ligand interactions at the molecular scale. Moreover, the possibility of tailoring the AFM probe with different chemical groups and individual molecules, coupled with the ability to

ty


carry out measurements under near - physiological conditions, made this approach ideal for the pharmaceutical and life sciences. 27 To date, most MRFS studies were performed either on receptors isolated on surfaces or using fixed or live cells. However, this approach is not representative of what happens in an in vivo setting.

The microenvironment of a cell is complex and is composed by other cells (either of the same origin or different), extracellular matrix components, fluids, and various molecular factors derived from these. The feedback loops of a cell and its surrounding are indispensable for cellular homeostasis and highly influences cell behavior, including the expression of certain molecules at the cellular membrane. 28

Taking this into consideration, the main goal of this study was to propose a methodology to assess the specificity of neuron - targeted NPs toward biological models of higher complexity and clinical relevance. For this, we exploited the presence of thiol groups at the surface of the previously developed neuron - targeted TMC - based NPs to stably attach them to an AFM tip and probe them against cell lines, heterogeneous primary cell cultures and histological tissue sections of clinically relevant sites of administration. As these NPs already proved to have neural tropism, they represent a good tool to validate MRFS throughout the different biological models under testing.

Our results show the selective binding and internalization of the Tg NPs toward the neuronal cell line (ND7/23 cells), in opposition to fibroblasts or Schwann cells ( $\mathrm{NIH}_{3} \mathrm{~T}_{3}$ or $\mathrm{RN} 22$ cells, respectively). More importantly, the Tg NP selectivity toward neuronal cells was reinforced when these were tested in a more complex cell culture system as the DRG primary cultures. Furthermore, in this model, a higher frequency of multiple unbinding events was observed on the primary DRG neurons, pointing to a higher abundance of $\mathrm{HC}$ receptors expression than on the ND7/23 cells. These results are in accordance to other studies that have reported a high expression level of the ganglioside family of receptors in DRGs, specially the GT1 b receptors-around $85 \%$ in small and $>90 \%$ in medium and large DRG neurons in rat, mice or human tissues (as determined by immunohistochemistry).29 Moreover, the expression of GTıb was found to be superior in the neuronal cell bodies and nerve termini, compared to peripheral nerve trunks.30 This could explain our results, as the data acquired for neuronal cells present in the DRG primary cultures was obtained from performing FDC in neuronal cell bodies. In contrast, the nT NPs presented a statistically significant lower BP, independently of being probed against neuronal or non - neuronal cells. Moreover, the data from confocal microscopy validate the obtained results from MRFS, where the selective binding of Tg NPs toward neuronal cells could be observed. Overall, this set of results validate the use of AFM to evaluate NP neuronal vectoring capacity in both cell lines and DRG primary cultures, and allowed us to obtain new insights into $\mathrm{HC}$ receptor expression levels in both systems.

As the ultimate goal of the development of this NP system is to obtain a vector to be administered in vivo via a minimal invasive route, we further evaluated the vectoring capacity of the neuron targeted TMC NPs in histological tissue sections of clinically relevant sites of administration. Our results showed similar results of binding probability and forces for FDC acquired in the sciatic nerve, when compared to the results obtained in neuronal cells from the primary DRG cultures. We would expect to have a slight increase in the binding probability as the binding of HC - QDs to this tissue sample is very abundant around the axonal extensions. However, when performing MRFS in a histological cryosection of the sciatic nerve, one has to consider that some regions of the nerve trunk present in the tissue section will be myelinated and, therefore, the neuron cell membrane will not be

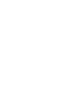


exposed. This also justifies the higher variance of binding probability values observed (ranging from 9.4 to $45.6 \%$ ). Nevertheless, in the innervated NMJ from gastrocnemius muscle sample, a statistically significant high percentage of unbinding events per tip was observed, confirming that this is a suitable tissue for the peripheral administration of the NPs. Also, our data show that in the NMJ the $\mathrm{HC}$ receptors density is very high as the majority of the FDC obtained in these specimens presented between 2 and 4 unbinding events, explained by the interaction of multiple $\mathrm{HC}$ moieties present at the surface of the probed NP. This can be either GT1b receptors or other types of gangliosides, which can also interact with the $\mathrm{HC}$ fragment. However, and since the binding forces are identical between each unbinding event, we believe that these unbinding events result from the higher density of the $G_{1} \mathrm{~b}$ receptors in this region. The specificity of this interaction was proven through the acquisition of FDC in the muscle, with both Tg and nT NPs, where a significant drop of the BP was observed.

We further determined the rupture forces between the HC or Tg NPs and the different biological models and, as expected, found a logarithmic dependence of the rupture force with increasing loading rates. This behavior is in accordance with a well - established theory for receptor-ligand interaction, 22 proving once more the specificity of the observed unbinding events. Moreover, no significant differences were found between rupture forces or the kinetics of ligand-receptor interaction between the tested NPs and the HC protein (independently of the model tested). This result assures that the neuron - targeting effect of the TMC - based NPs is being mediated by the $\mathrm{HC}$ molecules exposed at the NP surface through the used PEG spacer.

In summary, this biotechnological study shows the feasibility of the proposed method for the development and characterization of targeted nanomedicines in different biological models.

\section{Conclusion}

The development of techniques able to correctly evaluate NP vectoring capacity is in emergent need. Moreover, it is important to have a reliable method that allows reproducible and comparable results independently of the model being tested (isolated cell lines, heterogeneous cellular cultures or tissue samples) to fasten the development of these nanoparticulate systems. We believe that MRFS has the potential to be used as a screening and characterization tool that will fasten comparative investigations of different drug - delivery systems and targeting agents. Moreover, by combining fluorescent microscopy with AFM force spectroscopy one is able to correlate the specific interactions of the ligand-receptor pair in specific cells of heterogeneous primary cultures or cellular regions of histological sections. As this technology allows for the evaluation of native biological samples under physiological conditions, it could be used to assess cell targeting regardless of the desired application.

\section{Experimental Section}

Synthesis of Partially Thiolated TMC: TMC derived from ultrapure chitosan was supplied by Kytozyme (Belgium) (lot VIHAoo13 - 157) and purified by filtration and dialysis, as previously described.15, 31 Further details on polymer characterization were described in the Supplementary Materials and Methods (SMM) in the Supporting Information.

TMCSH was synthesized by the immobilization of thiol groups from 2 - iminothiolane on the primary amino groups of the polymer, as described elsewhere.12, 14 The resulting powders were stored at

(1) 
$-20{ }^{\circ} \mathrm{C}$ until further use. Determination of polymer thiol content was performed based on the Ellman's assay17 (see SMM in the Supporting Information).

Nanoparticle Preparation and Characterization: TMCSH - plasmid DNA NPs were prepared through self - assembly by mixing, while vortexing, equal volumes of pDNA and TMCSH solutions (in $20 \times$ 10-3 $\mathrm{m}$ HEPES buffered saline (HBS) with $5 \%$ (w/v) glucose solution, $\mathrm{pH} 7.4$ ). Nanocomplexes were prepared at a molar ratio of quaternized amine groups $(\mathrm{N})$ to moles of pDNA phosphate groups $(\mathrm{P})$ N/P molar ratio-of 8. TMCSH NP core (naked NPs; Figure $1 \mathrm{a}$ ) were let to stabilize for 15 min before further use or functionalization. For NP functionalization, TMCSH-pDNA nanocomplexes were incubated for $24 \mathrm{~h}\left(37^{\circ} \mathrm{C}, 1000 \mathrm{rpm}\right.$ ) with either a $5 \mathrm{kDa}$ MeO - PEG - MAL (Sigma - Aldrich) (nTNPs) (Figure 1 b) or with the PEG - modified HC fragment (Tg NPs) (Figure 1C). Details for the preparation of PEG - HC can be found in the SMM in the Supporting Information.

All formulations (naked, nT and Tg NPs) were characterized in terms of size, Pdl and zeta - potential using a Zetasizer Nano Zs (Malvern Instruments). The Smoluchowski model was applied for zeta potential determination and the cumulant analysis was used for average particle size determination. Ten micrograms of pDNA was used to prepare the tested formulations. All measurements were performed in triplicate, at $25^{\circ} \mathrm{C}$.

For pDNA condensation efficiency evaluation, the previously described SYBRTM Gold (Life Technologies, Thermo Fisher Scientific) exclusion assay was used.15 This assay enables the measurement of free PDNA, due to SYBRTM Gold binding to free nucleic acids (not complexed into NPs). Results are expressed as relative percentage of complexation, where $100 \%$ means pDNA is totally complexed with polymer in NPs, and o\% means that all pDNA is free (not complexed with the polycation). Samples with the same mass ratio of polymer without pDNA were used as control to subtract any polymer background fluorescence.

Internalization Studies in Cell Lines: ND7/23 (mouse neuroblastoma $\mathrm{N}_{18} \mathrm{tg} 2$ ) $\times$ rat dorsal root ganglion neuron hybrid), $\mathrm{NIH}_{3} \mathrm{~T}_{3}$ (mouse embryonic fibroblast), and RN22 (immature rat Schwann) cells were seeded into 24 well plates (Greiner bio - one), $24 \mathrm{~h}$ prior to incubation with NPs. TMC based NPs were prepared at N/P ratio of 8 using $2 \mu \mathrm{g}$ pDNA cm-2 and suspended in complete Dulbecco's modified Eagle medium (DMEM) (with serum and antibiotics). To assess the NP internalization profile, NPs were prepared as described above using YOYO ${ }^{\circledR}-1$ labeled pDNA. After an incubation period of $2 \mathrm{~h}$ in the presence of NPs, cells were incubated with trypan blue to quench any external fluorescence and subsequently trypsinized and processed for flow cytometry. Twenty thousand gated events were taken for each replicate and analyzed using a FACS Calibur flow cytometer (BD Biosciences). The resulting data was analyzed using FlowJo software (version 10, FLOWJO, LLC). Untreated cells were used as negative control.

Internalization Studies in DRG Primary Cultures: Primary dissociated DRG cells were seeded and cultured in complete neuron culture medium as mentioned in SMM. After $2 \mathrm{~d}$ in culture, TMC - based NPs loaded with $\mathrm{YOYO}^{\circledR}$ - 1 pDNA were added to the cells. Naked, $\mathrm{nT}$ or Tg NPs were prepared at an N/P ratio of 8 using $2 \mu \mathrm{g}$ pDNA cm-2 and suspended in complete neuron culture medium (with serum and antibiotics). After an incubation period of $2 \mathrm{~h}$, cells were washed with phosphate buffered saline (PBS) twice and subsequently fixed with $4 \%$ (w/v) PFA for 10 min at $37^{\circ} \mathrm{C}$. Afterward, a protocol 
for neuron discrimination was conducted (see SMM in the Supporting Information). For identification of cell nuclei, $100 \mu \mathrm{L}$ of a $2 \mu \mathrm{g} \mathrm{mL-1} \mathrm{HCS} \mathrm{CellMaskTM} \mathrm{Orange} \mathrm{Stain} \mathrm{(Molecular} \mathrm{Probes)} \mathrm{solution} \mathrm{was}$ added to each coverslip and incubated with cells for $30 \mathrm{~min}$ at RT, protected from light. After washing with PBS, cells were imaged using a laser scanning confocal microscope Leica TCS SP5 II (Leica Microsystems, Germany) with the HC PL APO Lbl. Blue $63 \times / 1.40$ oil objective. For each treatment group, a total of 20 fields were randomly selected for representative imaging. All images were acquired with the same laser intensity to allow for qualitative and semi - quantitative comparison between the different NPs profile of cellular association/uptake. Images were acquired from two independent experiments.

HC Conjugation with Quantum Dots: HC was conjugated to Qdot $705 \mathrm{~nm}$ ITK carboxyl quantum dots (Invitrogen), according to a previously described protocol.32 Briefly, $50 \mu \mathrm{L}$ of the Odot stock solution $(8 \times 10-6 \mathrm{~m})$ was diluted by adding $400 \mu \mathrm{L}$ of $0.01 \mathrm{~m}$ phosphate buffer, $\mathrm{pH} 8.5$ containing $20 \mu \mathrm{L}$ of 1 ethyl - 3 - (3 - dimethylaminopropyl)carbodiimide at $1 \mathrm{mg} \mathrm{mL}-1$, and mixed at RT for $10 \mathrm{~min}$ in the dark. $\mathrm{HC}(20.5 \mu \mathrm{L}$ of $3.3 \mathrm{mg} \mathrm{mL}-1$ stock solution) in $0.1 \mathrm{~m}$ phosphate buffer, $\mathrm{pH} 8$ was then added to the reaction mixture, thus giving a molar ratio of $3 \mathrm{HC}: 1 \mathrm{Odot}$. After $2 \mathrm{~h}$ of constant stirring at $\mathrm{RT}$ in the dark, the HC - Odots conjugate (hereafter designated as HC - ODs) solution was washed and concentrated using a ultracentrifugal filter with a $30 \mathrm{kDa}$ cut - off membrane (Millipore) to a final protein concentration of $1.5 \times 10-6 \mathrm{~m}$.

Identification of $\mathrm{HC}$ Receptor with $\mathrm{HC}-\mathrm{ODs}$ : $\mathrm{ND}_{7} / 23, \mathrm{NIH}_{3} \mathrm{~T}_{3}$, and $\mathrm{RN} 22$ cells were plated at a final density of $2.0,2.5$, and $2.0 \times 104$ viable cells $\mathrm{cm}-2$ on glass coverslips coated with poly(D - lysine) (PDL) and plated in 24 well plates. DRG primary cells were isolated and cultured as described above. After $48 \mathrm{~h}$, cells were incubated at $4^{\circ} \mathrm{C}$ for $10 \mathrm{~min}$ and then washed three times with cold PBS before a 30 min incubation with $\mathrm{HC}$ - ODs at a final concentration of $5 \mu \mathrm{g} \mathrm{mL}-1$ in $0.1 \%$ (w/v) BSA in PBS, at $4^{\circ} \mathrm{C}$. In the case of $\mathrm{ND} 7 / 23$ cells, a control was performed with a 10 min incubation of unlabeled $\mathrm{HC}$ (50 $\mu \mathrm{g} \mathrm{mL-1)}$ prior to the incubation with HC - ODs. Afterward, cells were washed with cold PBS three times and then fixed with $4 \%(\mathrm{w} / \mathrm{v})$ PFA prepared in PBS for $15 \mathrm{~min}$ at RT. After washing twice with cold PBS, cells were permeabilized for 5 min with Triton X - $1000.1 \%(v / v)$ prepared in PBS and incubated with $1 \%$ (w/v) BSA for $1 \mathrm{~h}$. All cell lines were then incubated with phalloidin 488 (Invitrogen) prepared in $1 \%(w / v)$ BSA solution (1:100) for 1 additional hour for cytoplasmic staining.

Tissue Section Preparation and Labeling with HC - ODs: Gastrocnemius muscles and sciatic nerves were collected from a young adult B6 mouse. Tissues were fixed in $4 \%(w / v)$ PFA overnight at $4{ }^{\circ} \mathrm{C}$ and cryopreserved in a $30 \%(\mathrm{w} / \mathrm{v})$ sucrose solution (Sigma - Aldrich). Cryosections of gastrocnemius muscles ( $50 \mu \mathrm{m}$ thick) and sciatic nerve ( $16 \mu \mathrm{m}$ thick) were obtained in a cryostat (HM550, Microm) and collected in $0.01 \mathrm{mg} \mathrm{mL-1} \mathrm{PDL} \mathrm{-} \mathrm{coated} 35 \mathrm{~mm}$ glass bottom dishes (10 $\mathrm{mm}$ microwell diameter, MatTek).

Frozen sections of gastrocnemius muscles were double stained for the postsynaptic marker $\alpha$ bungarotoxin and $\beta I I I$ tubulin, to allow the identification of NMJ. In brief, tissue sections were blocked for $1 \mathrm{~h}$ at RT with $5 \%(\mathrm{v} / \mathrm{V})$ normal goat serum in PBS containing $0.3 \%(\mathrm{v} / \mathrm{v})$ Triton $\mathrm{X}-100$ and incubated with the primary monoclonal antibody mouse anti - $\beta$ III tubulin (1:1000, Promega) overnight at $4{ }^{\circ} \mathrm{C}$. After washing in PBS, tissue sections were incubated for 90 min at RT with a secondary antibody rabbit anti - mouse Alexa Fluor 488 conjugate (Molecular Probes; 1:1000), $\alpha$ bungarotoxin-tetramethylrhodamine conjugate (Molecular Probes; 1:200) and HC - QDs, all diluted in blocking buffer. 
AFM Tip Functionalization: Silicon nitride AFM cantilevers with $0.01-0.03 \mathrm{~N} \mathrm{m-1}$ nominal spring constant (Bruker AFM Probes, MSCT) were amino functionalized as previously described, 33 using a gas - phase silanization method with (3-aminopropyl)triethoxysilane (APTES) (Sigma - Aldrich). The attachment of $\mathrm{HC}$ to the AFM tip was attained using a heterobifunctional PEG spacer, acetal PEG - NHS (PEG18) (Figure 2d), as previously described.34 Briefly, the aminofunctionalized tips were incubated for $2 \mathrm{~h}$ in $0.5 \mathrm{~mL}$ of a $6.6 \mathrm{mg} \mathrm{mL-1}$ acetal - PEG - NHS solution in chloroform (Sigma Aldrich) with $2 \%(\mathrm{v} / \mathrm{v})$ triethylamine (Sigma - Aldrich) at RT. After washing the tips three times in fresh chloroform and drying them in a N2 flux, the AFM tips were incubated for 10 min in $1 \%(v / v)$ citric acid (Sigma - Aldrich) in water to convert the acetal group into an aldehyde group. Afterward, the protein was coupled to the tips by incubating $100 \mu \mathrm{L}$ of $\mathrm{HC}$ in PBS at $0.2 \mathrm{mg} \mathrm{mL}-1$ containing a 2 $\mu \mathrm{L}$ of a $1 \mathrm{~m} \mathrm{NaCNBH} 3$ solution (Sigma - Aldrich). Then, ethanolamine hydrochloride solution ( $5 \mu \mathrm{L}$, $1 \mathrm{~m}, \mathrm{pH} 8$; Sigma - Aldrich) was added and the incubation proceeded for $10 \mathrm{~min}$ at RT. This procedure allows for the blockage of any unreacted aldehyde groups. Finally, the functionalized tips were rinsed three times with filtered PBS and kept in this solution at $4^{\circ} \mathrm{C}$ until further use.

The functionalization of AFM tips with the developed TMC - based NPs was attained through the use of a MAL - PEG - NHS spacer (PEG18) (Figure 2e), as previously described.10

Molecular Recognition Force Spectroscopy Studies: All measurements were carried out on a PicoPlus 5500 AFM instrument (Keysight Technologies, USA). Force-distance cycles (approach-retraction cycles) were carried out at RT by using HC - or NP - functionalized AFM tips.

Force spectroscopy studies using ND7/23, $\mathrm{NIH}_{3} \mathrm{~T}_{3}$, or RN22 fixed cells ( $4 \%$ (w/v) PFA, 20 min) cultured on PDL - coated glass coverslips and mounted on a standard fluid cell on the AFM scanner were performed in PBS at RT. Force-distance cycles were recorded at a $2000 \mathrm{~nm} \mathrm{s-1}$ pulling rate. The loading rate was varied via the rate of piezo expansion (pulling speed in $z$ - direction), which was adjusted by means of sweep duration time and scan range for trace and retrace. To provide an insight into the loading rate dependence of the $\mathrm{HC}$ or NPs on their cell receptors, pulling speeds from 50 to $4000 \mathrm{~nm} \mathrm{~s}-1$ were used. To evaluate the specificity of the measured events, surface receptor blockage was accomplished by incubating cells with free $\mathrm{HC}$ at $0.1 \mathrm{mg} \mathrm{mL-1}$ in PBS for $1 \mathrm{~h}$ prior acquisition of further FDC.

For the correct positioning of the functionalized AFM tips when performing MRFS studies on DRG primary cultures and histological tissue sections, a combined setup where the AFM sample stage (PicoPlus 5500) was mounted to the body of an inverted widefield fluorescence microscope (Axio Observer Z1, Zeiss) was used.

Dana Analysis of Force-Distance Cycles: For each dataset, at least 10 cells or 3 tissue samples were probed with several independent HC or NP functionalized AFM - tips. Up to 2000 force-distance cycles were recorded at each cell/tissue location and the tip position was altered a few hundred nanometers every 100 curves to ensure that the binding events were statistically appropriate. Data analysis (cantilever spring constants and force-distance cycle analysis) was performed using in house algorithms implemented in MATLAB (MathWorks). The spring constants of the cantilevers were determined by using the thermal noise method.35 Empirical force distributions of the rupture forces of unbinding events (PDF) were calculated as previously described.21 Unspecific adhesions were not valued as rupture events. 


\section{Acknowledgements}

The authors would like to acknowledge the Bioimaging Center for Biomaterials and Regenerative Therapies of INEB (b.IMAGE) for the support with atomic force and confocal microscopy, Centro de Materiais da Universidade do Porto (CEMUP) for NMR analysis and the Biointerfaces and Nanotechnology Service (INEB - i3S) for nanoparticle size and zeta - potential analysis. The work was financed by Portuguese funds through FCT (Fundação para a Ciência e a Tecnologia) in the framework of the projects UID/BIM/04293/2013, PTDC/CTM - NAN/115124/2009, and PTDC/CTM NAN/3547/2014. C.P.G. and C.D.F.L. acknowledge FCT for their Ph.D. scholarships SFRH/BD/79930/2011 and SFRH/BD/77933/2011, respectively.

\section{Conflict of Interest}

The authors declare no conflict of interest.

\section{REFERENCES}

[1] P. D. Reddy, D. Swarnalatha, Int. J. PharmTech Res. 2010, 2, 2025.

[2] G. Tosi, B. Ruozi, D. Belletti, Nanomedicine 2012, 7, 1113.

[3] a) A. Z. Wang, F. Gu, O. C. Farokhzad, Expert Opin. Biol. Ther. 2008, 8, 1063; b) D. F. Emerich, C. G. Thanos, Biomol. Eng. 2006, 23, 171.

[4] a) M. Arachchige, Y. Reshetnyak, O. Andreev, J. Biotechnol. 2015, 202, 88; b) P. Debbage, Curr. Pharm. Des. 2009, 15, 153; C) C. Curtis, M. Zhang, R. Liao, T. Wood, E. Nance, Wiley Interdiscip. Rev. Nanomed. Nanobiotechnol. 2017, 9, e1422.

[5] V. Kumar Khanna, ISRN Pharmacol. 2012, 2012, 571394

[6] S. P. Vyas, A. Singh, V. Sihorkar, Crit. Rev. Ther. Drug Carrier Syst. 2001, 18, 1.

[7] N. Kamaly, Z. Xiao, P. M. Valencia, A. F. Radovic-Moreno, O. C. Farokhzad, Chem. Soc. Rev. 2012, 41, 2971.

[8] C. Lamprecht, P. Hinterdorfer, A. Ebner, Expert Opin. Drug. Delivery 2014, 11, 1237.

[9] D. J. Muller, Y. F. Dufrene, Trends Cell Biol. 2011, 21, 461.

[10] H. Oliveira, M. Rangl, A. Ebner, B. Mayer, P. Hinterdorfer, A. P. Pêgo, Small 2011, 7, 1236.

[11] H. Oliveira, R. Fernandez, L. R. Pires, M. C. L. Martins, S. Simões,

M. A. Barbosa, A. P. Pêgo, J. Controlled Rel. 2010, 143, 350.

[12] C. D. Lopes, C. P. Gomes, E. Neto, P. Sampaio, P. Aguiar,

A. P. Pêgo, Nanomedicine 2016, 11, 3205.

[13] C. D. Lopes, H. Oliveira, I. Estevao, L. R. Pires, A. P. Pêgo, Int. J.

Nanomed. 2016, 11, 2675 .

[14] C. D. Lopes, N. P. Gonçalves, C. P. Gomes, M. J. Saraiva, A. P. Pêgo, Biomaterials 2017, 121, 83.

[15] C. P. Gomes, A. Varela-Moreira, V. Leiro, C. D. Lopes, P. M. Moreno, M. Gomez-Lazaro, A. P. Pêgo, Acta Biomater. 2016, 46, 129 . 
[16] H. Oliveira, L. R. Pires, R. Fernandez, M. C. L. Martins, S. Simões, A. P. Pêgo, J. Biomed. Mater. Res., Part A 2010, 95A, 801.

[17] G. L. Ellman, Arch. Biochem. Biophys. 1959, 82, 70.

[18] O. Germershaus, S. Mao, J. Sitterberg, U. Bakowsky, T. Kissel, J. Controlled Rel. 2008, 125, 145.

[19] a) K. Sinha, M. Box, G. Lalli, G. Schiavo, H. Schneider, M. Groves, G. Siligardi, N. Fairweather, Mol. Microbiol. 2000, 37, 1041; b) J. Herreros, G. Lalli, G. Schiavo, Biochem. J. 2000, 347, 199.

[20] P. Hinterdorfer, W. Baumgartner, H. J. Gruber, K. Schilcher, H. Schindler, Proc. Natl. Acad. Sci. USA 1996, 93, 3477.

[21] W. Baumgartner, P. Hinterdorfer, H. Schindler, Ultramicroscopy 2000, 82, 85.

[22] E. Evans, K. Ritchie, Biophys. J. 1997, 72, 1541.

[23] P. Hinterdorfer, Y. F. Dufrêne, Nat. Methods 2006, 3, 347.

[24] F. Kienberger, A. Ebner, H. J. Gruber, P. Hinterdorfer, Acc. Chem. Res. 2006, 39.

[25] E. Evans, K. Ritchie, Biophys. J. 1999, 76, 2439.

[26] A. C. Anselmo, S. Mitragotri, Bioeng. Transl. Med. 2016, 1, 10.

[27] F. Variola, Phys. Chem. Chem. Phys. 2015, 17, 2950.

[28] T. Ludwig, R. Kirmse, K. Poole, U. S. Schwarz, Pflugers Arch. 2008, 456, 29.

[29] Y. Gong, Y. Tagawa, M. P. T. Lunn, W. Laroy, M. Heffer-Lauc, C. Y. Li, J. W. Griffin, R. L. Schnaar, K. A. Sheikh, Brain 2002, 125, 2491.

[30] C. A. Massaad, G. Zhang, L. Pillai, A. Azhdarinia, W. Liu, K. A. Sheikh, Sci. Rep. 2015, 5, 15766.

[31] P. M. Moreno, J. C. Santos, C. P. Gomes, A. Varela-Moreira, A. Costa, V. Leiro, H. Mansur, A. P. Pêgo, Mol. Pharmacol. 2016, 13, 344 .

[32] S. Chakraborty, J. Fitzpatrick, J. Phillippi, S. Andreko, A. Waggoner, M. Bruchez, B. Ballou, Nano Lett. 2007, 7, 2618.

[33] A. Ebner, P. Hinterdorfer, H. Gruber, Ultramicroscopy 2007, 107, 922.

[34] L. Wildling, B. Unterauer, R. Zhu, A. Rupprecht, T. Haselgrubler, C. Rankl, A. Ebner, D. Vater, P. Pollheimer, E. E. Pohl, P. Hinterdorfer, H. J. Gruber, Bioconjug. Chem. 2011, 22, 1239.

[35] a) H.-J. Butt, M. Jaschke, Nanotechnology 1995, 6, 1; b) J. Hutter, J. Bechhoefer, Rev. Sci. Instrum. 1993, 66, 1868. 
Nanoparticle self-assembly

a

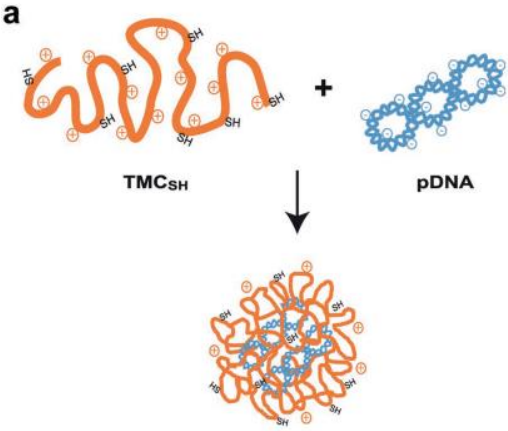

TMC-based Nanoparticles (naked NPs)

\section{Nanoparticle functionalization}

b

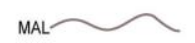

5 kDa MAL-PEG-MeO<smiles>C1=CCCCC=C1</smiles>

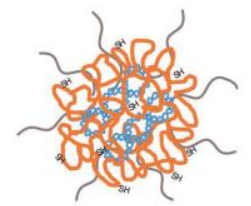

non-Targeted Nanoparticles (nT NPs)
C

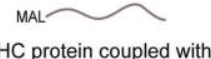

C protein coupled wit

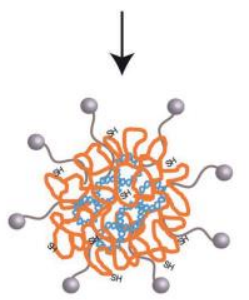

Targeted Nanoparticles (Tg NPs)
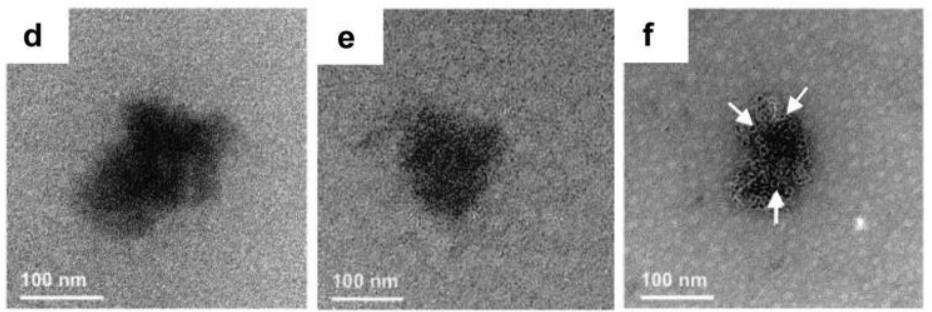

Figure 1. Preparation and characterization of trimethyl chitosan - based nanoparticles by transmission electron microscopy. Schematic representation for the preparation of trimethyl chitosan (TMC) nanoparticles: the core of the NPs was prepared by coacervation as a result of the electrostatic interaction between the a) polymer and plasmid DNA (naked NPs) and afterward NPs were functionalized either with b) a $5 \mathrm{kDa}$ maleimide (MAL) - PEG $\mathrm{MeO}$ chains (nT NPs), or with c) the HC protein coupled to a $5 \mathrm{kDa} \mathrm{N}$ - hydroxysuccinimide (NHS) - PEG - MAL spacer (Tg NPs). Transmission electron microscopy micrographs of suspended d) naked, e) nT, or f) Tg NPs samples. Tg NPs were prepared using HC - ODs (arrows) to distinguish the protein at the NP surface. 


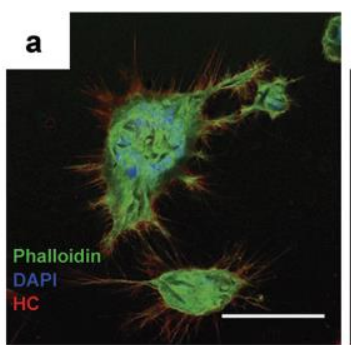

d

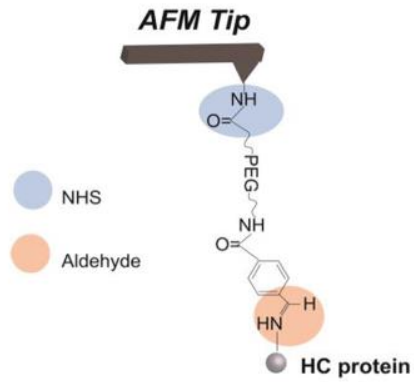

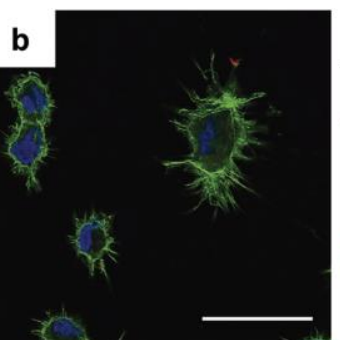

e
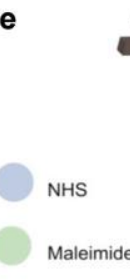

Maleimide

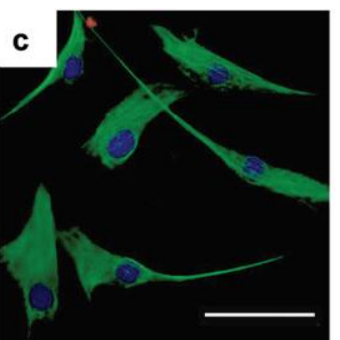

AFM Tip

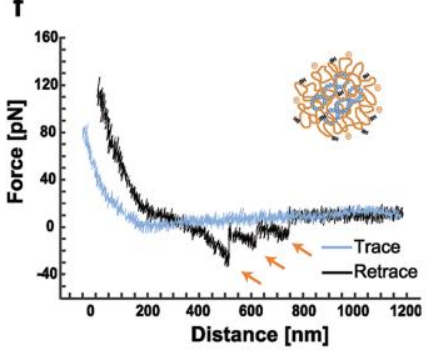

i

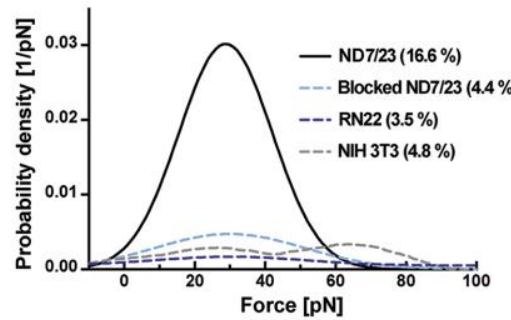

k

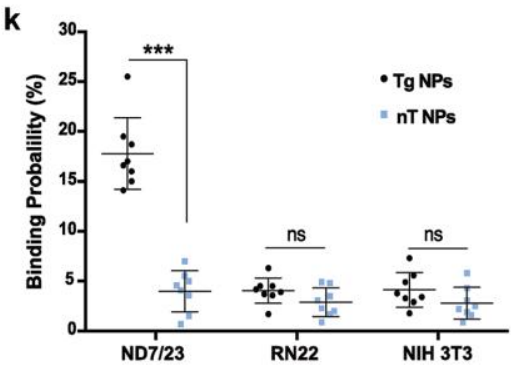

g

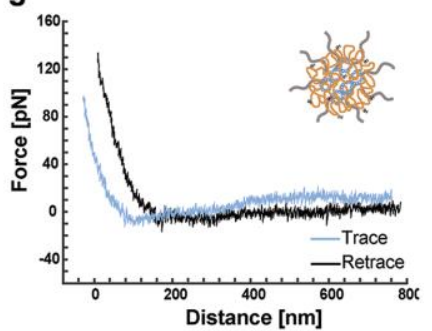

h

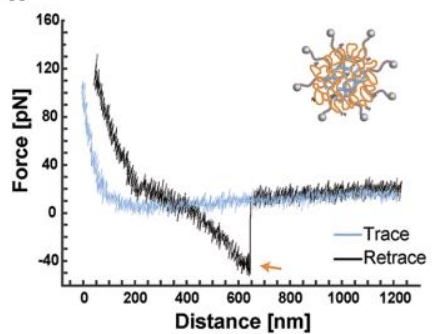

j
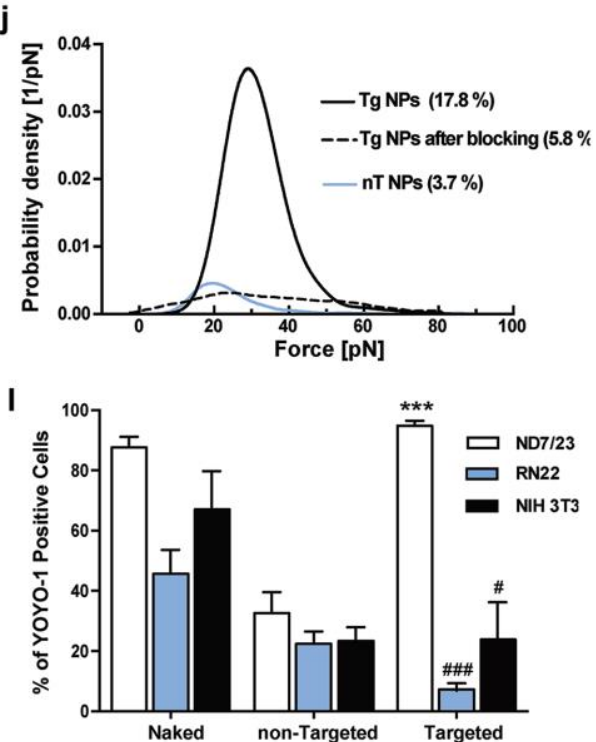

Figure 2. Evaluation of functionalized TMC - based nanoparticles specificity toward neuronal - like cells. Representative images of $\mathrm{HC}$ - ODs (red) binding to a) $\mathrm{ND}_{7} / 23$, b) RN22, and c) $\mathrm{NIH}_{3} \mathrm{~T}_{3}$ cells at $4{ }^{\circ} \mathrm{C}_{\text {; }}$ the cytoplasm and nuclei of cells were labeled with Phalloidin (green) and 4',6-diamidino - 2 - phenylindole (DAPI) (blue), respectively (scale bars: $25 \mu \mathrm{m}$ ). Schematic representation of the chemical tethering strategy used to bind d) the HC fragment or e) TMC - based NPs (naked, $\mathrm{nT}$ or Tg) to an AFM tip via heterobifunctional PEG spacers. 
Representative force-distance curves obtained in ND7/23 cells for $\mathrm{f}$ ) naked, g) nT, and h) Tg NPs; the presence of unbinding events is denoted by the orange arrays. The probability density function (PDF) of rupture forces showing specificity proof for i) HC - and j) NP - functionalized AFM tips; controls were performed by surface blocking with free $\mathrm{HC}$ ( $0.1 \mathrm{mg} \mathrm{mL}-1)$ for $1 \mathrm{~h}$; the unbinding probability for each condition is presented inside parentheses. $k$ ) Comparison between the binding probability of $\mathrm{nT}$ and $\mathrm{Tg}$ NPs in neuronal (ND7/23) and non - neuronal (RN22 and $\mathrm{NIH}_{3} \mathrm{~T}_{3}$ ) cell lines, each dot representing a different data set, corresponding to $1000-2000$ force-distance curves. The nonparametric multiple $t$ - test analysis was used for comparisons between both formulations in the different cell lines: $* * * p<0.001$. l) Percentage of YOYO ${ }^{\circledR}$ - 1 positive cells after incubation with TMC - based NPs containing labeled plasmid DNA for $2 h$; each bar represents the mean of three independent experiments $\pm S D$; the nonparametric multiple $t$ - test analysis was used for comparison between formulations: ${ }^{* * *} p<0.001$ for comparison between $\mathrm{nT}$ and Tg NPs; \#p $<0.05$ and \#\#\#p<0.001 when comparing naked with Tg NPs.

\section{.}



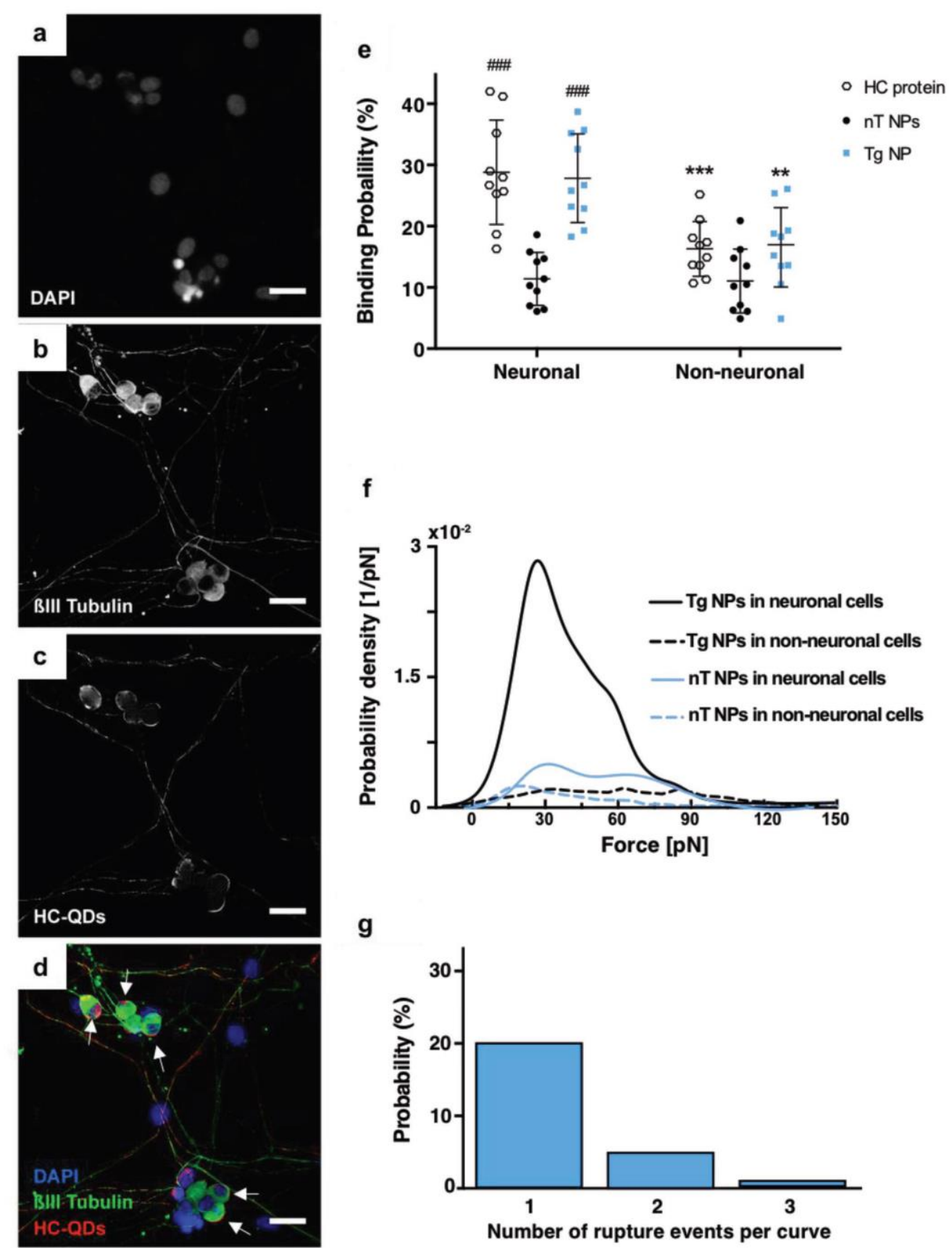

Figure 3. Binding specificity of functionalized TMC - based nanoparticles in primary dissociated dorsal root ganglia (DRG) cultures. Representative images for the HC binding pattern in DRG primary cultures; a) nuclei from all cells present in culture labeled with DAPI, b) neuronal cells stained for $\beta$ III Tubulin and c) HC - ODs binding pattern after incubation for $30 \mathrm{~min}$ at $4{ }^{\circ} \mathrm{C} ; \mathrm{d}$ ) all merged channels, with nuclei (blue), DRG neurons (green) and HC - QDs labeling (red; arrows); scale bars: $25 \mu \mathrm{m}$. e) Comparison between the binding probability of AFM tips functionalized with HC, nT or Tg NPs in neuronal versus non - neuronal cells present in the DRG cultures; each dot represents a different data set, corresponding to 1000-2000 force-distance curves. The nonparametric multiple t test analysis was used for comparisons between formulations in the different cell lines: \#\#\#p<0.001, when comparing $\mathrm{HC}$ or Tg NPs to the nT NPs in neuronal cells; $* * p<0.01$ and $* * * p<0.001$, when comparing each 
formulation in different cell types (neuronal versus non - neuronal). f) Probability density function (PDF) of rupture forces for AFM tips functionalized with $\mathrm{nT}$ and Tg NPs in different cell types. g) Representative binding statistics of one neuronal cell probed with an AFM tip functionalized with Tg NPs, where the total binding probability was $26.7 \%$.
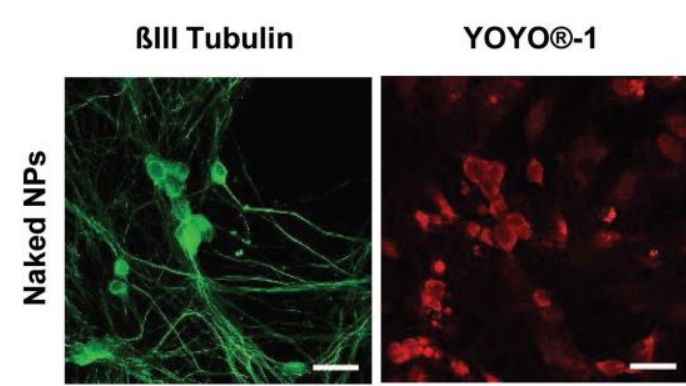
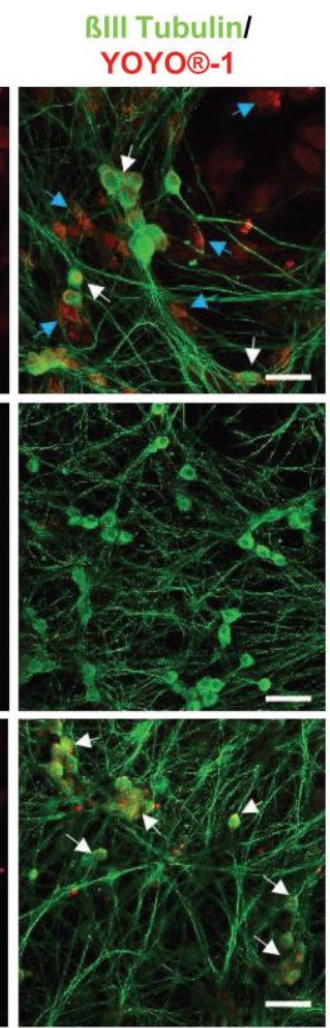

HCS CellMask $^{\mathrm{TM}}$

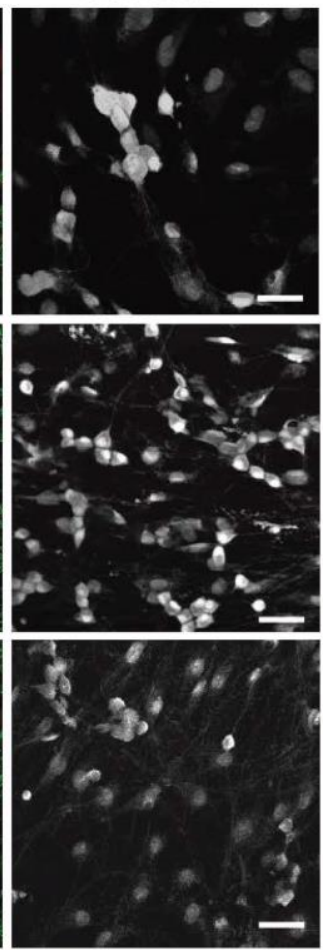

Figure 4. Cellular association of TMC - based nanoparticles to primary dissociated DRG cultures. Representative images of DRG cells incubated with naked, $\mathrm{nT}$ or Tg NPs loaded with YOYO ${ }^{\circledR}$ - 1 - labeled plasmid DNA. For neuronal cell identification, cells were immunostained for $\beta$ III tubulin (green). The NP cargo is identified in red (YOYO ${ }^{\circledR}$ - 1). HCS CellMaskTM (nucleus and cytoplasm stain) was used to identify all cells (neuronal and non neuronal) present in culture. The white arrows were used to identify NPs uptaken/associated to neuronal cells while the blue arrows label NPs uptaken/associated to non - neuronal cells. All images were acquired with the same laser intensity and contrast settings to allow for qualitative and semi - quantitative comparison between the different NPs profile of cellular association/uptake. Scale bars: $25 \mu \mathrm{m}$.
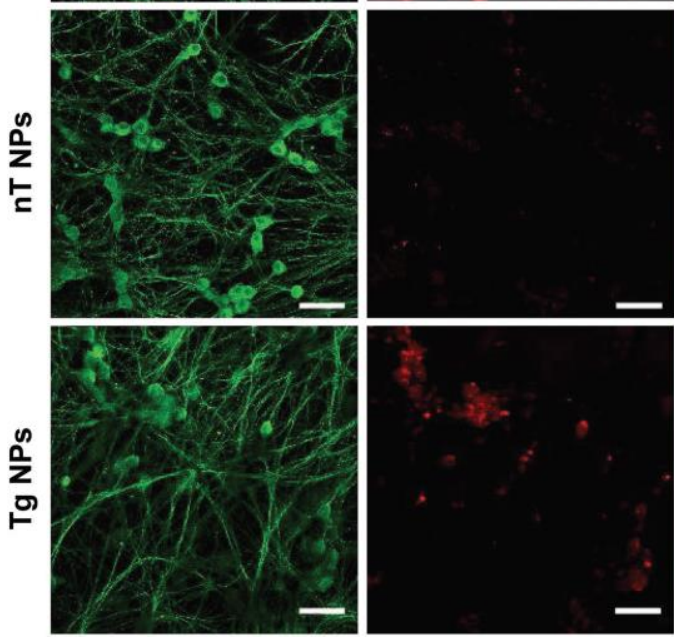
a
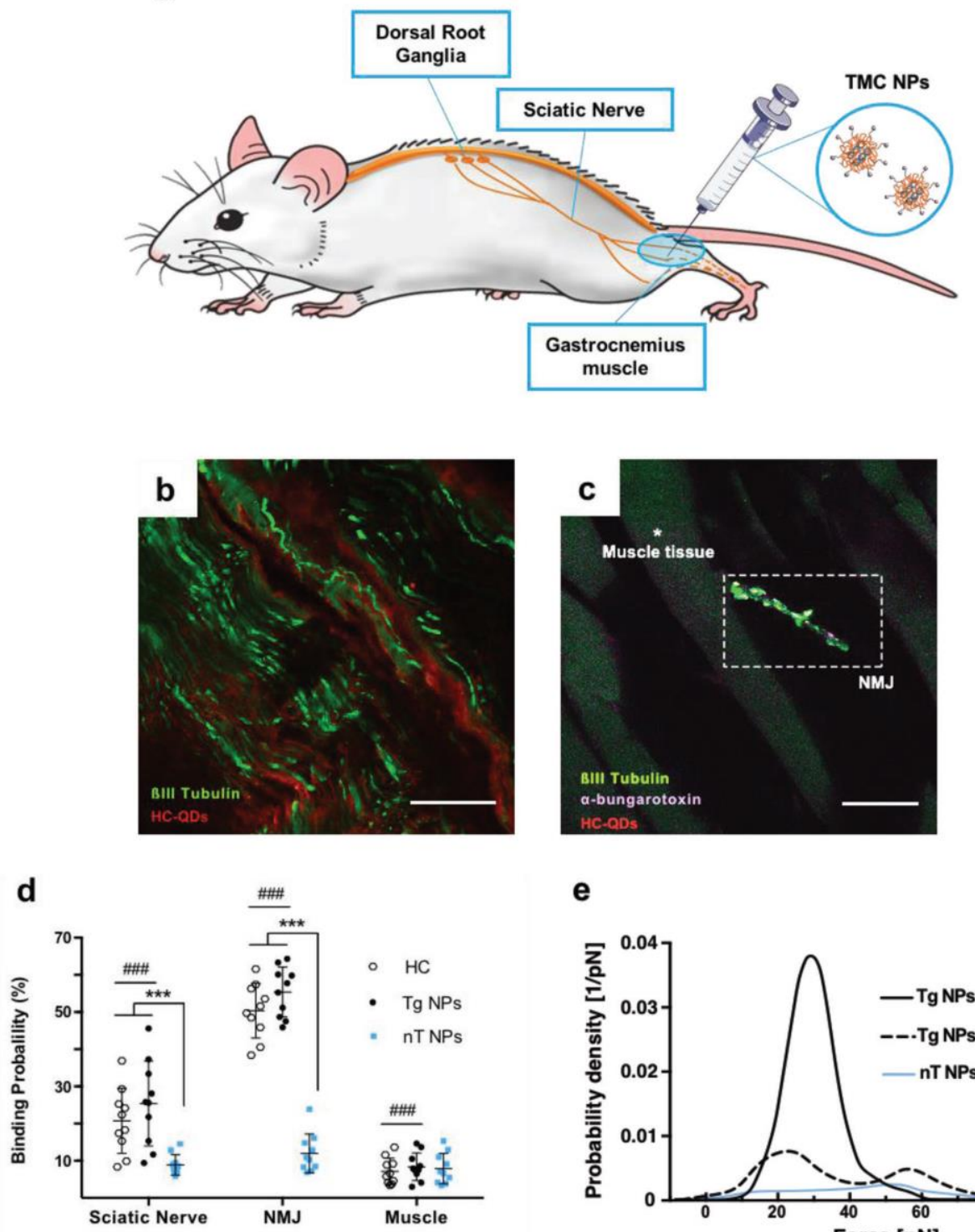

e

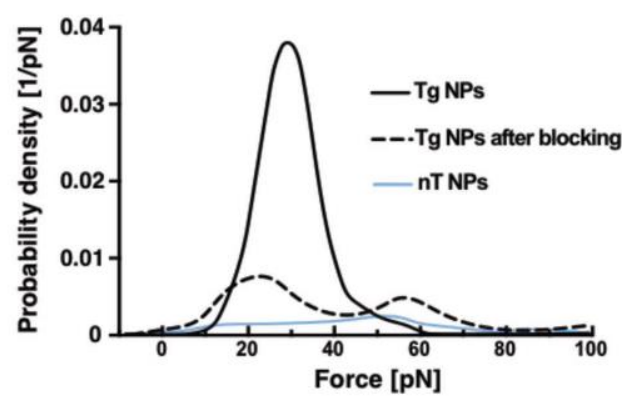

f

g
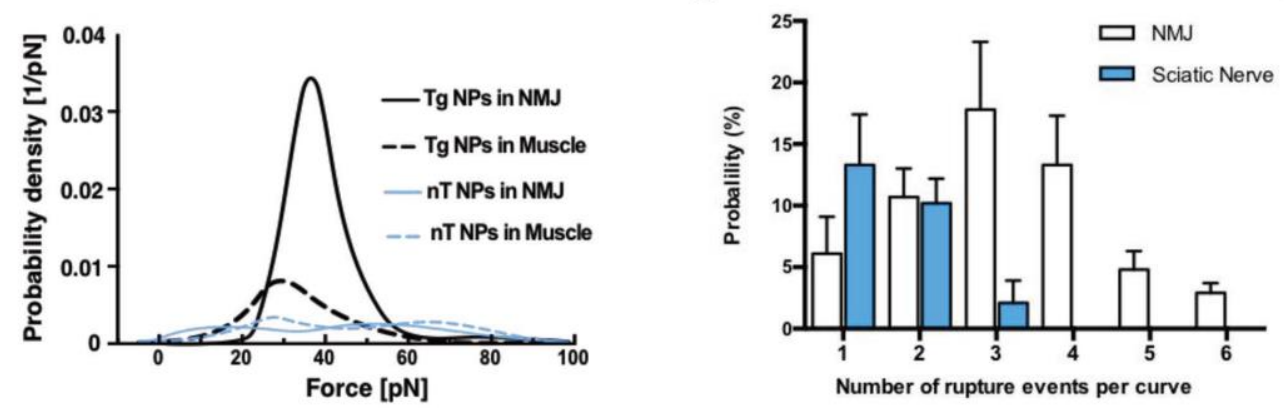

Figure 5. Binding specificity of functionalized TMC - based nanoparticles in histological tissue samples. a) Schematic representation of the proposed minimal invasive peripheral administration of the developed Tg NPs in 
an in vivo model. Representative images of HC - QDs binding pattern in tissue cryosections of b) sciatic nerve and c) gastrocnemius muscle; the frozen tissue sections were both labeled for $\beta$ III tubulin (green) for neuronal identification and the gastrocnemius muscles were further stained with the postsynaptic marker $\alpha$ - bungarotoxin (purple) to allow the identification of neuromuscular junctions (NMJ); HC - ODs labeling is identified in red; scale bars: $50 \mu \mathrm{m}$. d) Comparison between the binding probability of AFM tips functionalized with HC, nT or Tg NPs in specific cellular regions in the relevant histological sections, each dot representing a different data set. The nonparametric multiple $t$ - test analysis was used for comparisons between formulations in the different cell lines: $* * * \mathrm{p}<0.001$, when comparing the nT NPs binding probability to HC or Tg NPs in the same cellular region; \#\#\#p < 0.001 , when comparing $\mathrm{HC}$ and Tg NPs binding probability between different cellular regions (sciatic nerve vs NMJ vs muscle). Probability density function (PDF) of rupture forces considering the interaction between the functionalized NPs and the e) sciatic nerve and ( $f$ ) gastrocnemius muscle tissue cryosections; controls were performed by surface blocking with free $\mathrm{HC}(0.1 \mathrm{mg} \mathrm{mL}-1)$ for $1 \mathrm{~h}$. 

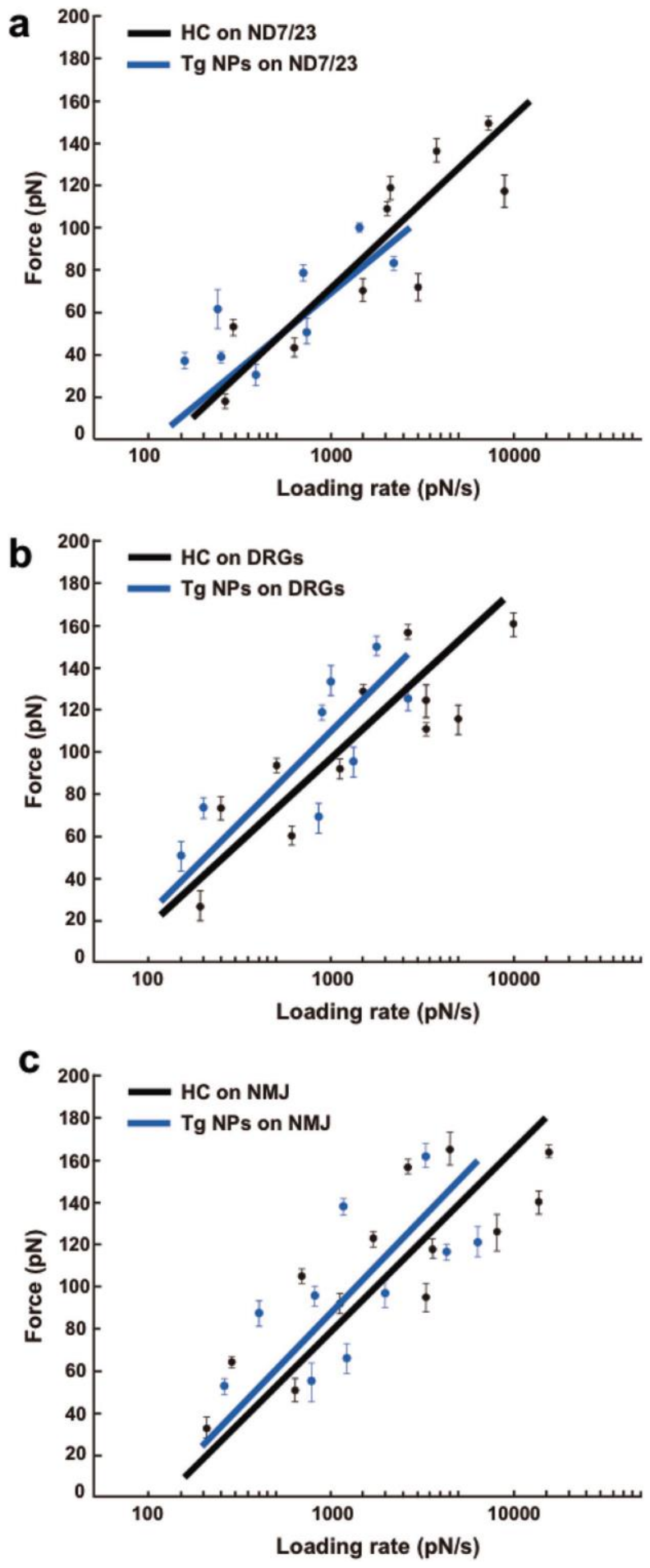

Figure 6. Loading rate dependence of $\mathrm{HC}$ fragment versus $\mathrm{HC}$ - functionalized TMC nanoparticles. Fitting of the measured unbinding forces at different loading rates considering the interaction between the HC fragment (black line) or Tg NPs (blue line) and a) ND7/23 cells, b) DRG primary cultures, and c) neuromuscular junctions (NMJ) in histological tissue sections. 
Table 1. TMC - based nanoparticles physicochemical characterization

\begin{tabular}{|c|c|c|c|c|}
\hline $\begin{array}{l}\text { Nanoparticle } \\
\text { formulation }\end{array}$ & $\begin{array}{c}\text { Size } \\
{[\mathrm{nm}]^{\mathrm{a})}}\end{array}$ & $\begin{array}{l}\text { Polydispersity index } \\
\text { (PdI) })^{\text {a) }}\end{array}$ & $\begin{array}{l}\text { Zeta-potential } \\
{[\mathrm{mV}]^{\mathrm{a})}}\end{array}$ & $\begin{array}{l}\text { pDNA condensation efficiency } \\
[\%)]^{\text {b) }}\end{array}$ \\
\hline Naked & $\begin{array}{c}222.0 \pm \\
8.1\end{array}$ & $0.281 \pm 0.037$ & $+21.5 \pm 0.5$ & $93.3 \pm 5.2$ \\
\hline non-Targeted & $\begin{array}{c}179.9 \pm \\
9.4\end{array}$ & $0.177 \pm 0.015$ & $+16.9 \pm 0.3$ & $90.8 \pm 7.4$ \\
\hline Targeted & $\begin{array}{c}167.3 \pm \\
11.2\end{array}$ & $0.219 \pm 0.029$ & $+19.4 \pm 0.2$ & $92.5 \pm 4.9$ \\
\hline
\end{tabular}

a)NPs average diameter size, Pdl, and surface charge (zeta - potential) were determined by dynamic light scattering in $20 \times 10^{-3} \mathrm{~m}$ HEPES buffered saline (HBS) with $5 \%(\mathrm{w} / \mathrm{v})$ glucose solution, $\mathrm{pH} 7.4$

b)pDNA condensation efficiency was determined by SYBR Gold exclusion assay in complete DMEM medium with serum and antibiotics, $\mathrm{pH} 7.4$.

Table 2. Kinetic parameters for HC fragment versus Tg NPs interaction with different biological models

$\begin{array}{lcc} & \mathbf{x}[\AA \AA \AA]^{\mathrm{a})} & \text { koff }[\mathbf{s - 1}]^{\mathrm{b})} \\ \mathrm{HC} \text { on ND7/23 } & 1.17 \pm 0.03 & 7.48 \pm 0.38 \\ \text { Tg NPs on ND7/23 } & 1.33 \pm 0.45 & 6.97 \pm 0.40 \\ \text { HC on DRGs } & 2.43 \pm 0.39 & 7.81 \pm 0.24 \\ \text { Tg NPs on DRGs } & 2.24 \pm 0.22 & 7.49 \pm 0.31 \\ \text { HC on NMJ } & 2.15 \pm 0.19 & 7.33 \pm 0.50 \\ \text { Tg NPs on NMJ } & 1.48 \pm 0.31 & 6.52 \pm 0.26\end{array}$

\footnotetext{
a) Energy barrier width

b) Kinetic off - rate.
} 